\title{
Ultrastructures of Germ Cells During Spermatogenesis and Taxonomic Values in Sperm Morphology in Male Mya arenaria oonogai (Heterodonta: Myidae)
}

\author{
Jin Hee Kim', Jae Seung Chung ${ }^{2}$ and Young-Je Park ${ }^{3}$ \\ ${ }^{1}$ Marine Eco-Technology Institute, Busan 608-830, Korea \\ ${ }^{2}$ Department of Urology, College of Medicine, Inje University, Busan 614-735, Korea \\ ${ }^{3}$ Sea Green Industry Institute, Bucheon 420-851, Korea
}

\begin{abstract}
The ultrastructures of germ cells during spermatogenesis and sperm morphology in male Mya arenaria oonogai, which was collected on the coastal waters of Samcheonpo, south coast of Korea, were investigated by transmission electron microscopic observations. In the early stage of the spermatid during spermiogenesis, a few granules and a proacrosomal granule, which is formed by the Golgi complex, appear on the spermatid nucleus, and then it becomes a proacrosomal vesicle. Consequently, it becomes an acrosome by way of the process of acrosome formation. The morphologies of the sperm nucleus type and the acrosome of this species have a curved cylindrical type and cone shape, respectively. The spermatozoon is approximately $48-50 \mu \mathrm{m}$ in length including a curved cylinderical sperm nucleus (about $2.65 \mu \mathrm{m}$ long), an acrosome (about $0.64 \mu \mathrm{m}$ in length) and tail flagellum (40-45 $\mu \mathrm{m}$ long). As some ultrastructural characteristics of the acrosomal vesicle, the peripheral parts of two basal rings show electron opaque part (region), while the apex part of the acrosome shows electron lucent part (region). These charateristics of the sperm belong to the family Myidae or some species of Veneridae in the subclass Heterodonta, unlike a characteristic of the subclass Pteriomorphia showing all part of the acrosome being composed of electron opaque part (region). Therefore, it is easy to distinguish the families or the subclasses by the acrosome structures. Exceptionally, In particular, a cylinder-like nucleus of the sperm is curved (the angle of the nucleus is about $20^{\circ}$ ), as seen in some species of Veneridae (range from $0^{\circ}-80^{\circ}$ ). The number of mitochondria in the midpiece of the sperm of this species are four, as one of common characteristics appeared in most species except for a few species in Veneridae in the subclass Heterodonta. Cross-sectioned axoneme of the sperm tail flagellum shows a 9+2 structure: the axoneme of the sperm tail flagellum consists of nine pairs of peripheral microtubules at the periphery and a pair of central doublets at the center.
\end{abstract}

Keywords: Mya arenaria oonogai, spermatogenesis, germ cell, sperm morphology

\section{INTRODUCTION}

Germ cell differentiation during spermatogenesis and mature sperm morphology have been documented to varying degrees in many species of bivalves using both light and electron microscopy (Franzén, 1970;

Received: November 30, 2011; Accepted: December 22, 2011

Corresponding author: Kim, Jin Hee

Tel: +82 (70) 8630-7109 e-mail: jhkim@marine-eco.co.kr $1225-3480 / 24416$
Daniels et al., 1971; Dorange and Le Pennec, 1989; Healy, 1989, 1995; Gaulejac, 1995; Chung and Ryou, 2000; Chung et al., 2005, 2006, 2007; Chung, 2006). It is well-known that spermatogenesis occurs various germ cell differentiations in the acini of the testis. Therefore, it is important to investigate the processes of germ cell differentiations by the developmental stages during spermatogenesis to clarify the reproductive mechanism.

To date, there are a few studies on unimodal cycle and bimodal cycle associated with the reproductive cycle and spawning cycle of M. arenaria (Ropes and 
Stickney, 1965; Brousseau, 1978), and spermiogenesis (Kim, 2001). Although the reproductive ecology of this species has been investigated by some authors, little information is available on germ cell differentiation during spermatogenesis and taxonomic values of mature sperm morphology and structure by electron microscopic observation in detail. In the Mollusca, sperm ultrastructure is considered a valuable tool in assessing taxonomic and phylogenetic problems within the Bivalvia (Franzén, 1970, 1983; Daniels et al., 1971; Popham, 1979; Healy, 1989, 1995; Koike, 1985; Hodgson and Bernard, 1986; Eckelbarger et al., 1990; Eckelbarger and Davis, 1996) and is especially useful when comparing closely related species (Popham et al., 1974; Popham, 1979).

In particular, it is well-known that acrosomal morphology of sperms has been used to organize bivalve subclasses (Popham, 1979), the number of mitochondria in the sperm midpiece tends to be stable within any given family or superfamily. For that reason, it needs to study acrosomal morphology of the sperm and the number of mitochondria in the sperm midpiece for taxonomic analyses of this species.

If some characteristics obtained from sperm ultrastructure and the process of spermatogenesis are phylogenetically analyzed, the results of the ultrastructural studies on bivalve spermatozoa will provide information needed for the elucidation of relationship patterns among several bivalve subclasses (Popham et al., 1974, Popham, 1979; Healy, 1989, 1995). For the study of taxonomic analysis of $M$. arenaria oonogai, which belongs to Myidae in the subclass Heterodonta, acrosomal morphology of mature sperm needs to compare their similarities with many species in other families of Heterodonta, and the number of mitochondria need to compare with many species in the families or superfamilies in the subclass Hetrodonta.

Therefore, the main aim of the present study is to describe the ultrastructural characteristics of germ cells by developmental stages, and to confirm sperm type with sperm ultrastructure by phylogenetic analysis of $M$. arenaria oonogai in Myidae in the subclass Heterodonta.

\section{MATERIALS AND METHODS}

\section{Sampling}

A total of 75 male specimens of $M$. arenaria oonogai were collected monthly in the intertidal zones of coastal waters of Samcheonpo, Gyeongsangnam-do, Korea, from January to December, 2006. The specimens were transported to the laboratory where they were maintained in seawater at $20^{\circ} \mathrm{C}$. The specimens were used for ultrastructural study of germ cells and mature spermatozoa by transmission electron microscopy.

\section{Transmission electron microscope observation}

For transmission electron microscope observations, excised pieces of the gonads were cut into small pieces and fixed immediately in $2.5 \%$ paraformaldehyde glutaraldehyde in $0.1 \mathrm{M}$ phosphate buffer solution $\left(\mathrm{pH}\right.$ 7.4) for 2 hours at $4^{\circ} \mathrm{C}$. After prefixation, the specimens were washed several times in the buffer solution and then postfixed in a $1 \%$ osmium tetroxide solution in $0.2 \mathrm{M}$ phosphate buffer ( $\mathrm{pH}$ 7.4) for 1hour at $4{ }^{\circ} \mathrm{C}$. Specimens then were dehydrated in increasing concentrations of ethanol, cleared in propylene oxide and embedded in an Epon-Araldite mixture. Ultrathin sections of Epon-embedded specimens were cut with glass knives on a Sorvall MT-2 microtome and LKB ultramicrotome at a thickness of about $80-100 \mathrm{~nm}$. Tissue sections were mounted on collodion-coated copper grids, doubly stained with uranyl acetate followed by lead citrate, and observed with a JEM 100 CX-II (80-KV) electron microscope.

\section{RESULTS}

\section{Ultrastructure of germ cells during spermatogenesis}

Based on the testicular development and morphological characteristics of germ cells by electron microscopic observations, in general. spermatogenesis occurs in the acini of the testis, and for convenience. The reproductive cycle can be divided into four stages: (1) spermatogonia, (2) spermatocytes, (3) spermatids, and (4) spermatozoa. In general, the process of spermatogenesis of this species appears to be similar to those of other bivalve species. 
Spermatogonia: Primary spermatogonia are located along the internal wall of the acini. In the first layer, spermatogonia are sometimes present near the accessory cells. Spermatogonia are approximately 6.2-7.3 $\mu \mathrm{m}$ in size and outlines of the cells are more or less oval-shaped. Each of primary spermatogonia contains a large nucleus with chromatin. The nucleus is about $4.3 \mu \mathrm{m}$ in diameter and has a patch appearance due to the arrangement of chromatin. Several mitochondria are distributed in the cytoplasm. Primary spermatogonia divide mitotically to produce secondary spermatogonia, which are smaller cells with smaller nuclei compared to primary spermatogonia. At this time several mitochondria are present in the cytoplasm (Fig. 1).

Spermatocytes: The secondary spermatogonia differentiate into primary spermatocytes (5.4-6.5 $\mu \mathrm{m}$ in diameter) by mitotic division. Two stages of spermatocyte development are present: the primary and secondary spermatocytes. The nucleus (3.2-3.6 $\mu$ $\mathrm{m}$ in diameter) of the primary spermatocyte contains slightly denser chromatin than that of the spermatogonium. The pachytene stage is characterized by the presence of synaptonemal complexes in the nucleus. The synaptonemal complexes in the nucleus appear in the prophase during the first maturation division.

Several mitochondria appear in the cytoplasm. Several secondary spermatocytes and several mitochondria in the cytoplasm. The primary spermatocytes differentiate into secondary spermatocytes by the secondary maturation division. At this time, the nucleolus is no longer prominent. The secondary spermatocytes are rarely observed, probably due to the rapidity of the second meiotic division of the primary spermatocytes. Their nuclei (about $3 \mu \mathrm{m}$ in diameter) are irregular in shape and the heterochromatin materials in the nucleus of the secondary spermatocyte are denser and more highly concentrated than those of the primary spermatocytes (Fig. 2).

Spermatids: The secondary spermatocyte is transformed into the spermatid by the secondary meiotic division. For convenience, spermiogenesis of spermatid has been divided arbitrarily into two stages: the early and late stages.

In the early stage of spermatids during spermiogenesis, spermatids are oval in shape and range from approximately $3-4 \mu \mathrm{m}$ in diameter. Their round nuclei (3.0-3.1 $\mu \mathrm{m}$ in diameter) contain scattered marginal heterochromatin and several mitochondria appear in the cytoplasm of the spermatid.

The morphology of the spermatid nucleus changes gradually during the differentiation of the spermatid. At this time, small granules are formed by the Golgi complex in the cytoplasm move to a position just in front of the nucleus, while mitochondria move to a position just behind the nucleus. At this time, the morphologies of the spermatid nuclei were gradually elongated, and one or a few granules appear on the nucleus of spermatid (Fig. 3). Thereafter, they become a proacrosomal vesicle on the nucleus (Fig. 4). The proacrosomal vesicle migrates to the presumptive anterior end of the spermatid nucleus, where, they coalesce to form a single electron-dense vesicle (Fig. 5). A con-like proacrosomal vesicle gradually becomes a round proacrosome. Thereafter, a proacrosme lying on the sperm nucleus becomes round cone in shape, and several mitochondria are reduced its number at the posterior part of the nucleus. However, the nucleus of the spermatid is still not curved (Fig. 6).

Thereafter, A round proacrosomal vesicle lied on the anterior nuclear fossa, the protrusion of a process begin from the anterior nuclear fossa part toward the upper part of the acrosomal vesicle. In the late stage of spermatid during spermiogenesis, especially, two large mitochondria at the midpiece part appear beneath the nucleus (Fig. 7).

At this stage, the acrosomal vesicle of spermatid is formed, and the acrosomal vesicle is composed of electron dense part (region) and electron lucent part (region): especially, the basal and lateral parts of the basal rings of the acrosomal vesicle show electron dense part (region), while the anterior apex part of the acrosomal vesicle shows electron lucent part 


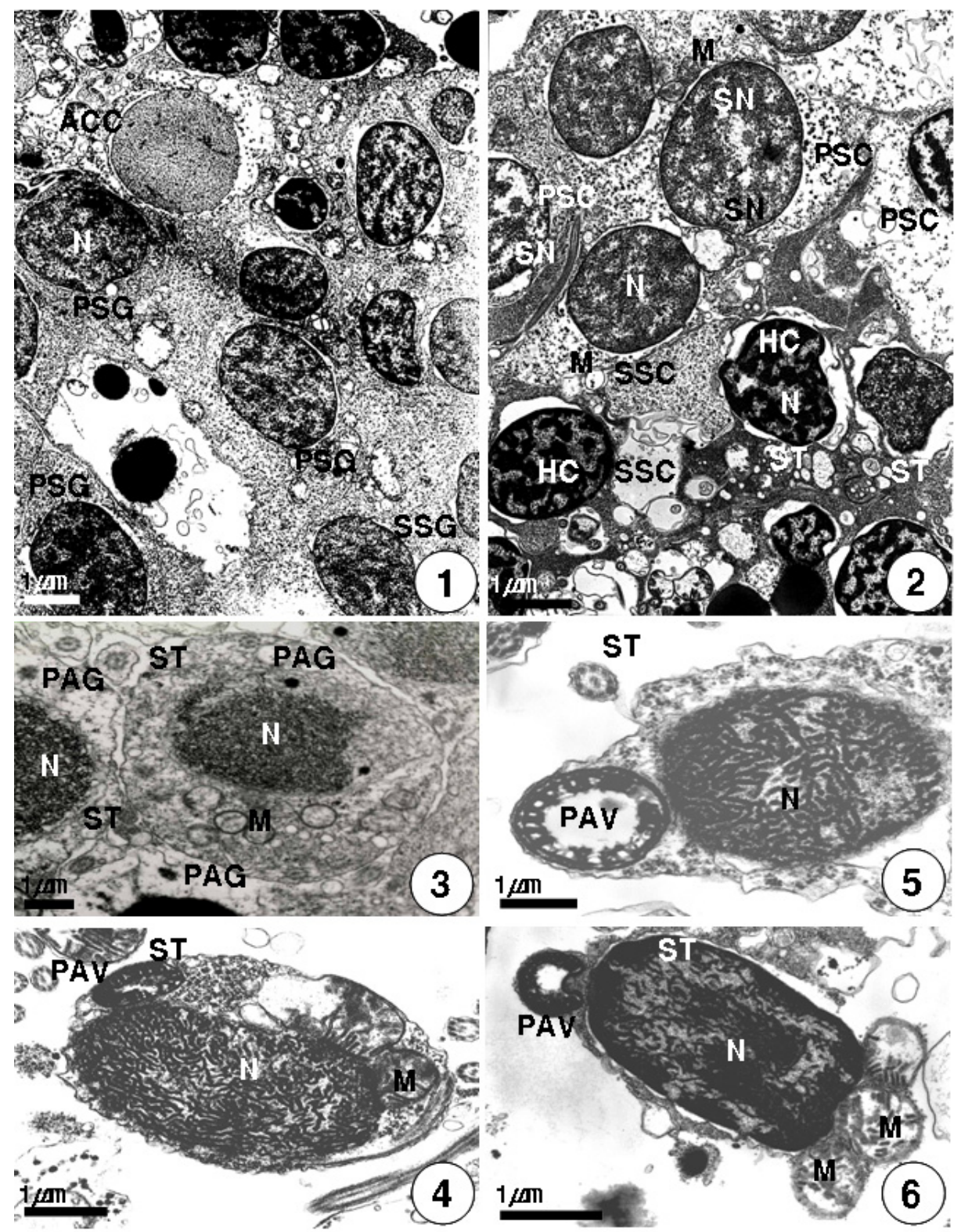

Figs. 1-11. Transmission electron micrographs of spermatogenesis in male Mya arenaria oonogai.

Fig. 1. Primary (PSG) and secondary spermatogonia (SSG). Note an accessory cell (ACC) and spermatogonia containing nuclei $(\mathrm{N})$ and several mitochondria $(\mathrm{M})$ in the cytoplasm.

Fig. 2. Primary spermatocytes (PSC), secondary spermatocytes (SSC) and spermatids (ST). Note several synaptonemal complexes (SN) in the nucleus of the primary spermatocytes (PSC) during the prophase of the first meiotic division, and heterochromatins (HC) in the nuclei of secondary spermatocytes (SSC), and spermatids (ST).

Fig. 3. Spermatids (ST) in the early stage of spermiogenesis. Note granules, proacrosomal granules (PAG) on the nucleus (N) of the spermatid.

Fig. 4. A spermatid (ST) in the same stage of spermiogenesis. Note a proacrosomal vesicle (PAV) on the nucleus (N) and mitochondria (M) in the early stage of the spermatid.

Fig. 5. A proacrosomal vesicle (PAV) and the spermatid during spermiogenesis. Note a proacrosomal vesicle just before the spermatid nucleus $(\mathrm{N})$.

Fig. 6. A round proacrosomal vesicle (PAV) on the nucleus $(\mathrm{N})$ of a spermatid (ST) during spermiogenesis. Note a proacrosomal vesicle (PAV), the elongated nucleus $(\mathrm{N})$ and spherical mitochondria $(\mathrm{M})$ beneath the nucleus $(\mathrm{N})$. 


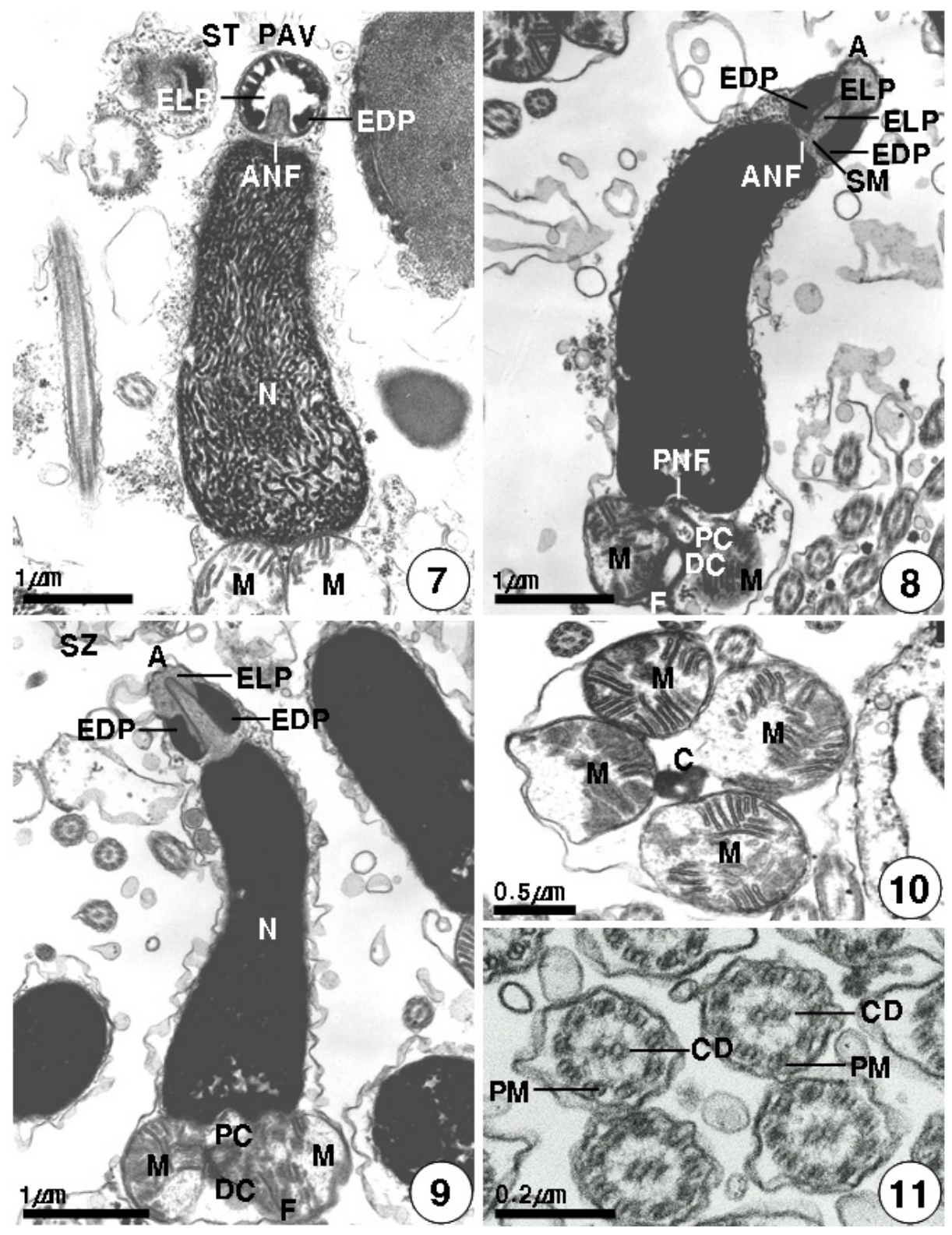

Fig. 7. A spermatid (ST) in the late stage of spermiogenesis. Note an proacrosomal vesicle being composed of electron dense part (EDP) and electron lucent part (ELP) on the anterior nuclear fossa of the elongated nucleus (N) on the nucleus $(\mathrm{N})$ of spermatid.

Fig. 8. Formation of the acrosomal vesicle containing subacrosomal material (SM) in the acrosome of a spermatid at the same stage. Note an acrosomal vesicle of the acrosome being composed of electron dense part (EDP) and electron lucent part (ELP), the anterior nuclear fossa (ANF) and posterior nuclear fossa (PNF) of the curved nucleus, the proximal (PC) and distal centrioles (DC), two spherical mitochondria $(M)$ and flagellum $(F)$.

Fig. 9. A completed spermatozoon (SZ) with an acrosome $(A)$, curved sperm nucleus $(N)$, the sperm midpiece and a flagellum (F). Note an acrosomal vesicle (AV) of the acrosome being composed of electron dense part (EDP) and electron lucent part (ELP), subacrosomal materials (SM), the anterior (ANF) and posterior nuclear fossas (PNF), the proximal $(P C)$ and distal centrioles (DC), two spherical mitochondria (M), and a flagellum (F).

Fig. 10. A cross sectioned sperm midpiece. Note a pair of centrioles (C) surrounding with 4 mitochondria (M).

Fig. 11. A cross sectioned sperm tail flagellum. Note the axoneme of the sperm tail flagellum showing a 9+2 structure (nine peripheral microtubules (PM) and a pair of central doublets $(C D))$. 
(region), as seen in characteristics of spermatozoa in the species and the families in the subclass Heterodonta. The axial filaments are not found in the acrosomal lumen, while subacrosomal granular materials are present in the subacrosomal space between the anterior nuclear fossa and the acrosomal vesicle of the acrosome. At this time, the acrosomal vesicle is composed of well developing basal rings, and the curved nucleus (angle of the nucleus: $20^{\circ}$ ) is covered with the plasma membrane. The anterior nuclear fossa exist in front of an acrosomal vesicle and the posterior nuclear fossa is present near the proximal centriole and distal centriole with some spherical mitochondria in the sperm midpiece.

At this time, of the two centrioles at right angles, show the classic nine triplets of microtubles. The proximal one lies in the posterior nuclear fossa and is perpendicular to the axoneme. It appears unconnected to the nuclear envelope. The distal centriole occupying the basal portion of the flagellum constitutes the basal body of the flagellum. The cytoplasm is greatly reduced, and so the nucle-cytoplasm is high (Fig. 8).

Spermatozoa: After spermiogenesis, the differentiation of spermatozoa are completed. Sperm morphology is primitive, as found in most bivalve species that undergo external fertilization. As some characteristics of the acrosomal vesicle structures in an acrosome of this species, the basal and lateral parts of the basal rings show electron dense part (region), while the anterior apex part of the acrosomal vesicle shows electron lucent part (region). These characteristics of the acrosomal vesicle were found in Myidae in the subclass Heterodonta.

Mature spermatozoa of this species measure approximately 48-50 $\mu \mathrm{m}$ long, and consist of conical acrosome positioned at the top of an elongated nucleus, a pair of centrioles surrounded by a ring of four spherical mitochondria, and a flagellum. The morphology of the sperm nuclear type and the acrosomal shape of this species are of a cylindrical type and a cone shape, respectively. The sperm head is about $3.97 \mu \mathrm{m}$ long and comprises a long, electron-dense nucleus (about $2.65 \mu \mathrm{m}$ long), with a anterior nuclear fossa and an acrosome. The acrosomal vesicle is about $0.64 \mu \mathrm{m}$ long membranebound, and deeply invaginated. The acrosomal vesicle is a cone shape. The morphology of the sperm nucleus and the acrosomes of this species are of a curved cylindrical type (angle of the nucleus is $20^{\circ}$ ) and a cone shape, respectively (Fig. 9). The proximal centriole and distal centriole surrounded with four spherical mitochondria with well-defined cristae appear in the sperm midpiece (Fig. 10). However, the axial filaments or an axial rod and satellite fibers are not found in the ultrastructure of mature spermatozoa of this species. And the tail flagellum is approximately 40-45 $\mu \mathrm{m}$ in length. A cross-sectioned tail flagellum shows that the axoneme is composed of a classic $9+2$ microtubular substructure (nine pairs of peripheral microtubules at the periphery surrounding a pair of central doublets at the center) enclosed by a plasma membrane (Fig. 11).

\section{DISCUSSION}

\section{Spermatogenesis}

In general, the testis of bivalves is composed of a number of acini, and spermatogenesis occurs in acini of the testis. The processes of germ cell differentiations and mature sperm ultrastucture of $M$. arenaria, were similar to those in the species in bivalves (Eckbarger and Davis, 1996; Chung et al., 2010). Therefore, the processes of spermatogenesis of bivalves was similar to those in the species in bivalves (Sakker, 1984; Bernard and Hodgson, 1985; Chung, 2006; Chung et al., 2007, Kim et al., 2010 a,b).

It is well-known that the processes of spermatogenesis occur through the interaction between germ cells and accessory cells in the acini. During spermatogenesis, the nucleus of the primary spermatocyte contains slightly denser chromatin than that of the spermatogonium. In this study, synaptonemal complexes appeared among scattered chromatins in the patchtene stage of the prophase during the first maturation division in the nucleus of the primary spermatocyte. Therefore, it shows a characteristics of the primary spermatocytes. the pachytene stage is 
characterized by the presence of synaptonemal complexes in the nucleus. In general, the main processes of spermatogenesis are morhological changes from spermatogonia to spermatozoa.

Yasuzumi (1974) described that the nuclei of spermatids can be classified into three morphological types according to the concentration morphology and degree of chromatins in the nuclei of spermatids: (1) granular, (2) fibrous, and (3) layer types. In general, in case of bivalves, it was a granular type in the early stage of chromatin concentrations, however, as the concentration of chromatins progresses, it was changed to be the fibrous type.

According to the morphologies of the sperm nuclei, the types of the sperm nuclei are of the cylinder type in M. lusoria, C. sinensis and Notochione jedoensis, the long cylinder type in Gomphina veneriformis, and the curved cylinder type in Ruditapes philippinarum, Saxidomus purpuratus, Dosinorbis japonicus and Mercenaria stinpsoni (Kim, 2001). In this study, the type of the sperm nucleus of $M$. arenaria oonogai belongs to the curved cylinder type.

This species belongs to external fertilization species and the type of spermatozoon is the primitive type. In particular, Kim (2001) reported that several species of Veneridae in subclass Heterodonta have three kinds of sperm nuclear types according to the angle of curved sperm nucleus: (1) no curved nuclear type (angle of the nucleus: $0^{\circ}$ ), (2) slightly curved sperm nuclear type (angle of the curved sperm nucleus: $5^{\circ}-10^{\circ}$ ), and (3) largely curved sperm nuclear type (angle of the curved nucleus: $15^{\circ}-80^{\circ}$ ). He reported that the angles of the sperm nucleus of the species in family Veneridae ranged from $0^{\circ}$ (Notochione jedoensis) to $80^{\circ}$ (Mercenaria stinpsoni).

In this study, its angle of the nucleus of $M$. arenaria oonogai in Myidae in subclass Heterodona was about $20^{\circ}$. Therefore, exceptionally, of the species in families in subclass Heterodonta, it is assumed that only Myidae and Veneridae species have the curved sperm nucleus.

In this study, the size of sperm nucleus of this species in Myidae was $2.65 \mu \mathrm{m}$ long. Kim (2001) reported that the sizes of sperm nuclei in Veneridae species ranged from $2.13 \mu \mathrm{m}$ (C. sinensis) to $7.80 \mu \mathrm{m}$ in diameter ( $G$. veneriformis). Therefore, he described that the morphologies of the sperm nucleus can not be used in taxonomic analyses because of irregular morphological characteristics of the nuclei (Healy, 1995).

It is well-known that the acrosome is formed by various granular secreations secreted by the Golgi complex (Longo and Dornfeld, 1967; Sastry, 1979). In Mytilus coruscus, several small proacrosomal vesicle are formed by the Golgi complexes, and these vesicles were mixed with each other in the acrosomal vesicle during spermiogenesis (Kim et al., 2010a). Eckelbarger and Davis (1996) reported that commonly proacrosomal vesicles were found in $C$. virginica spermatocytes. In this study, however, in $M$. arenaria oonogai a proacrosomal vesicle appeared spermatid stage and this vesicle developed to an acrosomal vesicle, and became an acrosome. The mechanism of acrosomal vesicle formation in mollusc sperm are diverse and that no single mechanism characterizes bivalve sperm.

In general, morphologies of proacrosomal vesicles showed some different speciality with the species, therefore, it is assumed that proacrosomal vesicles are formed in different course of most bivalves.

\section{Taxonomic values in mature sperm morphology and ultrastructure}

Sperm ultrastructure of bivalves is considered a valuable tool in assessing taxonomic and phylogenetic problems within the bivalvia (Franzén, 1970, 1983; Popham, 1979; Eckelbarger et al., 1990), and it is now widely used in taxonomic analyses (Healy, 1995): for example, 1) acrosomal morphology, and 2) the number of mitochondria in the sperm midpiece. Of sperm ultrastructures of bivalves, acrosomal morphology are now widely used in taxonomic analyses (Healy, 1995) because its morphological characteristics has been used to organize bivalve subclasses (Popham, 1979). Regarding acrosomal morphologies, Healy (1989) reported that different subclasses of bivalves each have unique acrosomal morphologies.

Regarding the acrosomal morphologies of the 
sperms, Kim (2001) reported that the acrosome can be classified into four shapes (cone, long cone, modified cone, cap, modified cap shape). The acrosomal morphologies of the sperms in Veneridae were the cone shapes in $C$. sinensis and D. japonicus, and a modified cone shape in $R$. philippinarum, and the cap shapes in Saxidomus japonicus, M. lusoria, Mercenaria stinpdoni, Notochione jedoensis. In this study, the acrosomal morphology of this species in Myidae has a cone shape, as seen in the family Veneridae.

Compared those of $M$. arenaria oonogai with the morphology of the sperm nuclei and the acrosomal vesicles in some species of Veneridae, the morphological or phylogenetical characteristics of this species are the presence of sperm nucleus and acrosomal vesicles showing the curved sperm nucleus and cone shape during spermatogenesis. Therefore, these are common characteristics between this species and some species of Veneridae. Accordingly, we assume that the presence of a special acrosomal vesicle during spermatogenesis could be used as a key characteristic for identification of species of the genus Mya in Myidae in subclass Heterodonta.

In particular, regarding a common structural characteristics of the acrosomal vesicles of the subclasses Heterodonta and Pteriomorphia in the bivalves, Hodgson and Bernard (1986) reported that all species in subclass Heterodonta in the bivalves have a common structural characteristics of the acrosomal vesicles showing the cone shape, and being composed of electron high density (opaque) material and electron lucent material. Hodgson and Bernard (1986) described that the acrosomes of the Heterodonta are characterized by restriction of the electron-dense material to the base or lateral regions, with such area joined by the acrosome membrane only. However, the subclass Pteriomorphia in the bivalves have a common structural characteristics of the acrosomal vesicles showing the cone shape, and being composed of electron high density (opaque) materials in all parts (Hodgson and Bernard, 1986).

In this study, taxonomically, $M$. arenaria oonogai belongs to the family Myidae in subclass Heterodonta
(Min et al., 2004). The acrosomal vesicles of this species is composed of electron high density (opaque) part (from the basal ring part to the lateral parts) and electron lucent part (the apex part (region)) as reported by Hodgson and Bernard (1986). Therefore, from morphologies of the acrosomal vesicles of the acrosome mentioned above, this species belongs to subclass Heterodonta reported by Hodgson and Bernard (1986).

Accordingly, these phenomena of this species showed remarkably similar patterns to various kinds of species of Mactridae, Veneridae, Tellinidae, Solenidae, Hiatellidae in subclass Heterodonta. As shown in Fig. 8 or 9, the nucleus and acrosomal morphologies of this species in Myidae in subclass Heterodonta were the curved cylinder type and cone shape. This point is one of common structural characteristics of the acrosomal vesicle, as shown in subclass Heterodonta. In particular, the anterior apex part of acrosomal vesicles of this species was very thin, and the boundaries of the inner and outer membranes were closely attached and acrosomal vesicles in acrosomes showed the cone shape, as shown in common characteristics of the subclass Heterodonta.

Regarding the number of mitochondria in the sperm midpiece, some authors (Hodgson and Bernard, 1986; Healy, 1989) stated that the number of mitochondria in the sperm midpiece tends to be stale within any family or superfamily varying from a maximum of 14 in the mytilord Modiolus difficilis (Drozdov and Reunov, 1986) to a minimum of 4 (Healy, 1989, 1995).

In this study, the number of mitochondria in the sperm midpiece of $M$. arenaria oonogai was four (common to many bivalve families), as seen in Solenidae, Corbiculidae in subclass Heterodonta and Ostreidae in subclass Pteriomorphia, while those of mitochondria were five in Arcidae, Mytilidae in subclass Pteriomorphia and some species of Veneridae (S. purpuratus, M. lusoria, Cyclina sinensis) and Atrina pinnata japonica). Because the number of mitochondria in the sperm midpiece showed stable and a constant characteristics under the level of 
family or in superfamily. Accordingly, the results of the number of the mitochondria are coincided with those reported by Hodgson and Bernard (1986) and Healy (1989).

In this study, the axial rod and satellite fibers are not found in mature sperm of this species in Myidae. Judging from the results on the ultrastructure of mature spermatozoon, it is supposed that this species belongs to subclass Heterodonta because the axial rod and satellite fibres are not found in the structures of the acrosomal vesicle of the spermatozoon, unlike the appearance of structures of the satellite fibres and acrosomal vesicles of Ostreidae and Mytilidae sperms (containing the axial rod) in subclass Pteriomorphia.

\section{ACKNOWLEDGEMENTS}

The authors are grateful to Prof. Emeritus, Ee-Yung Chung, Kunsan National University, and Dr. Hee-Woong Kang, West Sea Fisheries Instittute, NFRDI, for helpful comments and arrangement on the manuscript. This research was supported in part by the fund from the Research Projects of Sea Green Industry Institute, Bucheon, Korea.

\section{REFERENCES}

Bernard, R.T.F. and Hodgson, A.N. (1985) The fine structure of the sperm and spermatid differentiation in the brown mussel Perna perna. South Africa Journal of Zoology, 20: 5-9.

Brousseau, D.J. (1978) Spawning cycle, fecundity and recruitment in a population of soft-shell clam, Mya arenaria from Cape Ann, Massachusetts. Fisjeries Buletin, 76: 155-166.

Chung, E.Y. and Ryou, D.K. (2000) Gametogenesis and sexual maturation of the surf clam Mactra venerifermis on the west coast of Korea. Malacologia, 42: 149-163.

Chung, E.Y. (2006) Ultrastructure of germ cells during spermatogenesis and the reproductive cycle in male Meretrix petechialis on the west coast of Korea. The Korean Journal of Malacology, 22: 115-124.

Chung, E.Y., Kim, H.J., Kim, J.B. and Lee, C.H. (2006) Changes in biochemical components of several tissue in Solen grandis, in relation to gonad developmental phases. The Korean Journal of Malacology, 22: 27-38.

Chung, E.Y., Kim, E.J. and Park, G.M. (2007) Spermatogenesis and sexual maturation in male Mactra chinensis (Bivalvia: Mactridae) of Korea. Integrative Bioscience, 11: 227-234.
Daniels, E.W., Longwell, A.C., Mc Niff, J.M. and Wolfgang, R.W. (1971) A reinvestigation of the ultrastructure of the spermatozoa from the american oyster Crassostrea virginica. Trans American Microscopy Society, 90: 275-282.

Dorange, G. and Le Pennec, M.L. (1989) Ultrastructural characteristics of spermato- genesis in Pecten maximus (Mollusca, Bivalvia). Invertebrate Reproduction Development, 15: 109-117.

Drozdov, T.A. and Reunov, A.A. (1986) Spermatogenesis and the sperm ultrastructure in the mussel. Modiolus difficillis. Tsitologiia, 28: 1069-1074.

Eckelbarger, K.J., Bieler, R., and Mikkelsen, P.M. (1990) ltrastructure of sperm development and mature sperm morphology in three species of commensal bivalves (Mollusca: Galeommatoidea). Journal of Morphology, 205: 63-75.

Eckelbarger, K.J. and Davis, C.V. (1996) Ultrastructure of the gonad and gametogenesis in the eastern oyster, Crassostrea virginica. II. Testis and spermatogenesis. Marine Biology, 127: 89-96.

Franzén, A. (1970) Phylogenetic aspects of the mophology spermatozoa and spermiogenesis. In; Baccetti B (ed) "Comparative spermatology.". Accademia Nationale Dei Lincei, Rome, pp. 573.

Franzén, A. (1983) Ultrastructural studies of spermatozoa in three bivalve species with notes on evolution of elongated sperm nucleus in primitive spermatozoa. Gamete Research, 7: 199-214.

Gaulejac, de J., Jenry, M. and Vicente, N. (1995) An ultrastructural study of gametogenesis of the marine bivalve Pinna nobilis (Linnaeus, 1758). II. Spermatogenesis. Journal of Molluscan Studies. 61: 393-403.

Healy, J.M. (1989) Spermiogenesis and spermatozoa in the relict bivalve genus Neotrigonia: relevance to trigonioid relationships, particularly Unionoidea. Marine Biology, 103: 75-85.

Healy, J.M. (1995) Sperm ultrastructure in the marine bivalve families Carditidae and Crassatellidae and and its bearing on unification of the Crasssatelloidea with the Carditoidea. . Zoological Science, 24: 1-28.

Hodgson, A.N. and Bernard, R.T.F. (1986) Ultrastructure of the sperm and spermatogenesis of three species of Mytilidae (Mollusca, Bivalvia). Gamete Research, 15: $123-135$.

Kim, J.H. (2001) Spermatogenesis and comparative ultrastructure of spermatozoa in several species of Korean economic bivalves (13 families, 34 species). Pukyung National University 161 pp.

Kim, J.H., Chung, E.Y., Choi, K.H., Park, K.H. and Park, S.W. (2010a) Ultrastructure of germ cells during spermatogenesis and some characteristics of sperm morphology in Male Mytilus coruscus (Bivalvia: Mytilidae) on the west coast of Korea. The Korean Journal of Malacology, 26: 33-43.

Kim, J.H., Chung, E.Y., Choi, K.H., Lee, K.Y. and Choi, 
M.S. (2010b) Ultrastructure of the testis and germ cell development during spermatogenesis in male Crassostrea gigas (Bivalvia: Ostreidae) in western Korea. The Korean Journal of Malacology, 26: 235-244.

Longo, E.J. and Dornfeld, E.J. (1967) The fine structure of spermaid differentiation in the mussel, Mytilus edulis, L. Ultrastructural Research, 20: 462-480.

Min, D.K., Lee, J.S., Ko. D.B. and Je, J.G. (2004) Mollusks in Korea. Hanguel Graphics. 566 pp.

Popham, J.D. (1974) Comparative morphometrics of the acrosomes of the sperms of externally and internally fertilizing sperms of the sperms of the shipworms (Teredinidae, Bivalvia, Mollusca). Cell Tissue Research, 150: 291-297.

Popham, J.D. (1979) Comparative spermatozoon morphology and bivalve phylogeny. Malacological Review, 12: 1-20.
Ropes, J.W. and Stickney, A.P. (1965) Reproductive cycle of Mya arenaria in New England. Biological Bulletin, 128: 315-327.

Sakker, E.R. (1984) Sperm morphology, spermatogenesis and spermiogenesis of three species of chitons (Mollusca, Polyplacophora). Zoomorphology, 104: 111-121.

Sastry, A.N. (1979) Pelecypoda excluding Ostreidae. In Giese AC, Pearse JS eds. "Reproduction of Marine Invertebrates". Vol 5, New York. Academic Press pp. 113-292.

Verdonk, N.H., Van Den Biggelaar, J.A.M. and Tompa A.S. (1983) The Mollusca. Vol. 3. Development. Academic Press, New York, pp 48.

Yasuzumi, G. (1974) Electron microscope studies on spermatogenesis in various species. International Review Cytology, 37: 53-119. 